\title{
The neurobiology of language: Relevance to linguistics
}

\author{
E. Susan Duncan*1, 2, ${ }^{*}$, Sarah Tune ${ }^{2}$ and Steven L. Small ${ }^{1,2,3}$ \\ ${ }^{1}$ Department of Cognitive Sciences, University of California, Irvine, CA, USA \\ ${ }^{2}$ Department of Neurology, University of California, Irvine, CA, USA \\ ${ }^{3}$ Department of Neurobiology and Behavior, University of California, Irvine, CA, USA \\ ${ }^{4}$ Department of Communication Sciences \& Disorders, Louisiana State University, Ba- \\ ton Rouge, LA, USA \\ *duncan1@Isu.edu.
}

\begin{abstract}
The study of language is shared by a number of fields, including linguistics, psychology, and neurobiology. While the methods employed by these domains may overlap, they differ in the focus of their scientific inquiry, and the unique perspective of each may inform investigation within the others. We conceptualize this relationship in the context of David Marr's information processing theory, with neurobiology as the implementational level of language, and discuss the history of the neurobiology of language from early localizationist models to the present day.

Decades of electrophysiological and anatomical studies of the macaque monkey support the existence of dual streams for the processing of auditory information. More recent neuroimaging studies suggest that these streams are also present in humans, subserving speech perception and language comprehension. The development of high resolution brain imaging methods and brain stimulation has advanced our ability to study, in vivo, the structures and processes underlying the language network. For those linguists interested in studying language with consideration of the system that implements it, theories and concepts may now be meaningfully informed by neurobiology.
\end{abstract}

Keywords: psychology; information processing; dual stream model; human neuroscience; brain connectivity.

\section{What is the neurobiology of language?}

The earliest surviving scientific investigation of language is Panini's Sanskrit grammar dating from the fourth century BCE (Panini and Vasu, 1891). This complete and self-contained system of rules represents a thorough and considered inquiry into the structure of language, incorporating the work of pre- 
ceding scholars, which itself endures only through its citations. Panini's ancient text puts forth a comprehensive generative grammar that continues to inform theoretical and technical aspects of linguistics in the modern era (Kiparsky 1993).

In the ensuing millennia, linguistics has evolved and expanded to encompass myriad subfields devoted to various aspects of the scientific study of language. Among them are subdisciplines dedicated to understanding the structure and form of language, its historical evolution, and how it conveys meaning, as well as the dynamics of context-sensitive language use. Additionally, distinct lines of inquiry into the nature of language have emerged from newer disciplines. Psychology interrogates language as a uniquely human behavior that permits insight into underlying mental representations and processes of interest. Neurobiology is concerned with the physical composition of the nervous system, including neurons and their organization into circuits that permit complex behaviors such as language to occur.

The neurobiology of language emerges at the intersection of these three fields. It is characterized by a strong biological foundation that is complemented by our knowledge of the cognitive processes and linguistic functions it implements. Importantly, to advance our understanding of the relationship between the brain and language, it is imperative that each of the three disciplines provides critical elucidations and constraints on research and interpretation in the other two. The success of the modern neurobiology of language hinges on the cooperative efforts of researchers from different disciplines who not only share the same methods of inquiry and topic of scholarship, but who also build on common assumptions and work towards the goal of understanding how the brain "does" language. A first step towards this goal is to recognize that despite an overlap in subject matter, the research questions addressed by each field are fundamentally different. A clear differentiation of distinct levels of inquiry is a necessary prerequisite to understanding how these levels are linked to one another in the service of behavior.

In David Marr's influential posthumous text (Marr 1982), he identifies three levels requiring consideration in the quest to understand a complex information-processing system such as the human brain's ability to decode language. The first, and most abstract of these, is the computational or processing level, which examines the goal of the computation and the logic of the strategy for performing it. The second is concerned with the representation of both the input and the output of the process, as well as the algorithm that is applied for conversion between the two. The third level is the hard- 
ware implementation, or "nuts and bolts", explaining how this processing may occur in a physical sense. In terms of the triumvirate of linguistics, psychology and neurobiology, the domain-to-level mapping is clear. At the processing level lies linguistics, with its well-developed computational theories and logically ordered systems of rules. Psychology focuses on the mental objects and functions that might also be described as the representations and algorithms of Marr's second level. Finally, neurobiology's emphasis on the physical properties of the human nervous system straightforwardly poises it as the third level, implementation.

Marr believed these three levels of information processing in complex systems to constitute a rough hierarchy. The inherent linking between levels allows for some generalization across them, yet it is loose enough that no level defines any other. Therefore, one must exercise caution in interpreting evidence at one level as conclusions at another, and the best hypotheses will be those that not only incorporate all three levels but also focus on understanding the relationships among them. Neurobiology, as the biology of the nervous system, seeks to analyze the brain as a physical organ that processes and produces language through intrinsic connections with sensorimotor systems. However, any theory of the neurobiology of language that is solely focused on describing the physical properties of this underlying neural "hardware" would be incomplete. To describe the anatomy of the human brain, including its constituent neural cells and circuits, without incorporating the language functions it enables would be analogous to describing the anatomy of the heart and its muscle contractions without linking it to the flow of blood, transport of oxygen and nutrients, and removal of metabolic waste. Similarly, any computational model seeking to explain measurements of the shape, contractility, and subcomponent structure of the heart via echocardiography would have limited explanatory power if it did not consider the restrictions placed upon the observed function by the implementing organ.

In other words, irrespective of its starting point, any scientific inquiry into the neurobiology of language should aim at integrating the three levels of analysis. In line with these considerations, we define the neurobiology of language as: The brain implementation of representations and processes necessary and sufficient for production and understanding of speech and language in context. As such, it is our position that the ideal means for investigating the neurobiology of language is an interdisciplinary approach with contributions from each fundamental scientific field of the triumvirate, in addition to a clinician-scientist with expertise in human neuroscience, neuro- 
logical injury, degeneration, and repair, such as a neurologist or speechlanguage pathologist.

\section{Role of the neurobiology of language in linguistic theory}

Above we have highlighted the importance of interdisciplinary teamwork, including linguistic expertise, for a comprehensive neurobiology of language. The question of the role of the neurobiology of language in linguistics, conversely, is dependent on one's perspective on the nature of inquiry in linguistics. If linguistics is defined as the scientific investigation of language and its structure - as derived from the study of static corpora of output - then there is no need to incorporate psychology, neuroscience, or the neurobiology of language into one's analysis. If the definition encompasses more dynamic studies of linguistic behavior, then there may be need for psychology, but still no reason to include neurobiology. Thus, if one's aim is to study language (or languages) in the abstract, disembodied from the humans who use it, one need not venture past linguistics into other domains. Alternatively, if one wants to develop concepts in linguistics that consider or account for behavioral characteristics such as human performance, cross-linguistic or individual differences, pragmatics, or social context, then it is imperative to consider psychology. The reasons to incorporate biology must be above and beyond the structure of human languages and their use by human beings in the world, i.e., there must be an interest in the structure and function of the specific device - the human brain - that instantiates the implementation.

Models and theories that are restricted to the linguistic domain certainly serve a meaningful purpose. Their importance within the field of linguistics is undisputed, and the careful characterization of regularities and irregularities within and across languages can provide important constraints for the neurobiology of language. However, language is implemented in computations performed by the human brain. Thus, if one's aim is to develop linguistic concepts faithful to the system that produces and constrains the data on which they are based, then one needs to consider the nature of the component parts and their functioning. This means, for example, that an account of word or sentence processing would need to acknowledge existing research demonstrating that auditory and written language comprehension engage different sets of brain circuits (Buchweitz et al. 2009; Price 2012). Incorporating such interdisciplinary knowledge into a linguistic investigation represents a move towards a neurobiological approach to understanding language. 


\section{History of the neurobiology of language}

The origins of the neurobiology of language may be found in the work of nineteenth century European physicians. One of the earliest of these was Franz Joseph Gall in the first half of the 19th century. Gall interpreted bumps and depressions on the surface of the skull as indications of the development of the underlying neural tissue, and thus as evidence of the presence of personality traits attributable to the underlying brain region (Gall 1825). While Gall's phrenological methods have been rightly discredited as pseudoscience, he deserves credit for introducing the idea that various regions of the brain provide unique contributions to different forms of information processing. Phrenology served as a precursor to the cerebral localization of mental processes, including language, then thought to be situated behind the eye.

The next significant development was Pierre-Paul Broca's presentation of a case study of a patient with nonfluent aphasia before the Anthropological Society of Paris in 1861, in which he reported a lesion primarily occupying the posterior portion of the third convolution of the left frontal lobe (Broca 1861), citing the error of the phrenologists and declaring this area (now named Broca's area) to be the seat of spoken language. A decade later, Carl Wernicke expanded on Broca's findings by identifying that language disorders (i.e., aphasia) could be associated with damage to other left hemisphere brain regions in the vicinity of the Sylvian fissure (i.e., Wernicke's area), as well as damage to connecting association pathways (Wernicke 1874), pioneering the first of the connectionist theories of language. Further evolution of this idea was demonstrated by Lichtheim's "House" model (Lichtheim 1885), which elaborated the role of cortical connections and identified "disconnection" syndromes, Grashey's model of language processing (Grashey 1885), which identified distinct centers and connections for spoken vs. written language, and Déjerine's identification of the role of white matter connections in his writing on pure alexia without agraphia (Déjerine and Déjerine-Klumpke 1895). Freud objected to the conceptualization of language as a property arising from isolated centers and their interspersed connections, positing that the entire perisylvian cortex provided crucial, if heterogeneous, contributions to language (Freud 1891), although this work was largely ignored. In the mid-twentieth century, the Wernicke-Lichtheim model was resurrected by Norman Geschwind (Geschwind 1970), and it remains perhaps the predominant model in aphasia theory today. 
The importance of this work is paramount. Yet, as with the distinctions between the triumvirate fields informing our neurobiology of language, the types of questions we ask will always inform the types of answers we find. Lesion studies will invariably present us with localizationalist answers, as lesions are inherently localized. Similarly, many neuroimaging studies, particularly early studies, pinpoint circumscribed regions of activation correlated with language and other higher-order functions by using high activation thresholds, such that a single region might remain (e.g., Small et al. 1996). Many early studies that did not force such localized activation through statistical means nevertheless described their results in focal terms (e.g., Binder et al. 1994).

The "brain as a computer" is one common metaphor for characterizing the brain as the product of its localized abilities (Tooby and Cosmides 2005). By this conception, the brain is a collection of modular functions performed sequentially to achieve the desired output (Levelt 2001). Despite the graphical nature of their anatomical diagrams, this is not inconsistent with the views of the early aphasiologists, in which complex functionality is built through the combination of simple components. It is worthwhile to note here that the trend of associating brain function with the popular technology of the day is by no means new. Since the seventeenth century, the brain has been likened to a hydraulic system, a production factory, an electromagnetic device, a telegraph, a telephone switchboard, and, since the mid-twentieth century, a computer (Copeland 1993), all in keeping with the vanguard device of the times. The current notion of the brain as a network fits firmly into our contemporary society of Facebook and Twitter social interactions and airline hub and spoke systems.

Yet if the brain can be likened to a computer, it is not of the traditional type with hardware and software designed for the connection of separate modules for serial computation, executed on a single, or even multiple, central processing unit(s). With tens of billions of neurons, each with up to ten thousand connections (Agatonovic-Kustrin and Beresford 2000), the brain must instead be envisioned as a massively interconnected and parallel device. This realization gives rise to a conception of neural function that necessarily abandons classical models, with their modular constituents inexplicably summing to a profound level of complexity. Recognition of the physical realities of the brain must push our models forward.

Beyond localizationalism lies an appreciation of the brain as the product of overlapping and interconnected circuits. As our analytical methods ad- 
vance in tandem with our theoretical conceptualization, our investigations become more complex, reflective of the characteristics of the subject matter. Thus, as a field, we are now transitioning from strictly region of interest (ROI) analyses to the realm of connectivity and connectomics. In functional connectivity, the brain is typically understood through correlations between the time courses of anatomically separate regions, similarities of which are believed to reflect functional integration. The resulting connectivity measures commonly reflect average statistical dependencies collapsed across time, while more advanced analyses yield dynamic measures that express how functional connectivity varies as a function of time. In effective connectivity, a model is constructed to describe causal relationships among brain regions, often indicated by time-dependent variations of one region in relation to another. The term "connectomics" (Sporns et al. 2005), which originally referred to structural connections between neurons only and sought to build a full model of the brain's "wiring diagram", has since evolved to include functional models, as well as structural models constructed at a macroscale such as through the use of diffusion tensor imaging (DTI) (Goni et al. 2014). Additionally, graph theoretical analysis of neural networks, whether functional or structural, allows insights into organizational principles that govern the flow of information by modeling pairwise relationships among brain regions (Bullmore and Sporns 2009). Such quantitative methods, with their focus on interconnections rather than isolated regions, currently represent our strongest tools for investigations into the neurobiology of language in humans.

\section{Animal models of language}

The primary means of conducting neuroscience research is through the use of an animal model. In seeking to elaborate a neurobiology of language, this becomes somewhat problematic. As a uniquely human skill, language does not have a representative animal model such as might be found in the inquiry into other domains. Some studies use songbirds, which share some features with human language, such as non-imitative vocal learning and a critical period for development (Doupe and Kuhl 1999; Tchernichovski and Marcus 2014). However, birds are not close relatives of humans, being rather the last surviving dinosaurs. The most recent shared ancestor of birds and humans existed over 300 million years ago, and other animals closely related to either 
creature do not share similar vocal abilities. The extent to which humans and songbirds share similar features in this regard is the result of convergent evolution, in which disparate lineages independently develop mutually useful, but unrelated, traits. If we are to again reference Marr's levels of information-processing, it is clear that even if this coincidence of convergent evolution were able to inform our investigation into the computational and representational levels of analysis, it would be highly unlikely to provide any evidence for the implementation level. Therefore, the songbird as an animal model cannot inform our neurobiology of language as defined above.

If we are going to identify an animal model more productive for accomplishing a neurobiology of language, we would be wise to look at our closer relatives in the primate kingdom, particularly those with a demonstrated legacy of shared neural implementation of behavioral functions. It is theorized that our present system of oral communication developed after our ancestors' brains evolved to support imitation and pantomime, upon which we were eventually able to build a primitive system of protosign that ultimately evolved into the complex language system we use today (Arbib and Bota 2003). This emphasizes two important aspects of the phylogenetic roots of our communication system. The first is that our current communication behaviors are grounded in, and superimposed upon, the cognitive and sensorimotor abilities of our earlier primate ancestors. The second is that our earlier communication systems likely relied exclusively on manual gesture, and that the connection between gesture and language remains strong to this day.

One of our primate relatives, the macaque monkey, has an extensive history of use in neuroscience research. During investigation of the motor system, Rizzolatti et al. (di Pellegrino et al. 1992) found a subset of neurons firing in premotor cortex during observation of the experimenter's hand movements, despite the fact that the monkey itself remained motionless. These neurons - also active when the monkey moved the relevant hand movements - were labeled "mirror neurons" due to their apparent mirroring of observed actions. Further investigation revealed the presence of such neurons in frontal and parietal regions that were selectively active during the macaque's observation and execution of a given task requiring either hand or mouth movements (Ferrari et al. 2003; Gallese et al. 1996; Rozzi et al. 2008). In humans, functional magnetic resonance imaging (fMRI) acquired during observation of actions performed by the hand or mouth reveal activation in premotor cortices displaying a somatotopic pattern (e.g., with observation of 
mouth movements activating the same ventral areas active while producing mouth movements) (Buccino et al. 2001). This suggests that the organization and mirror properties of the macaque brain represent a reasonable model for the human cortical motor system.

The macaque is also an important animal model for understanding the visual system. Through electrophysiological study, cortical mapping, and targeted ablation, two separate cortical pathways, or streams, have been found to support the analysis of visual material (Ungerleider and Mishkin 1982). Both originate in the primary visual cortices, where low-level sensory information is processed. As information is transferred outside of primary visual cortices, the type of information that is processed becomes increasingly complex. One stream travels ventrally to inferior temporal regions and is critical for recognizing the defining features of an object required for its identification (i.e., the "what pathway"). The other stream travels dorsally through posterior parietal cortex carrying information relevant to motion and spatial qualities (i.e., the "where/how pathway"), facilitating the translation of purely sensory visual information into a motor plan for how to interact with the object perceived. Both visual streams ultimately end in prefrontal cortices, which play a unique role in decision-making and planning. Mapping between humans and monkeys suggests large-scale homology between the visual systems, even in higher order cortical regions (Orban et al. 2004).

Following the discovery of distinct pathways for the analysis of visual input in the macaque, investigators began to seek similar pathways from primary auditory to prefrontal cortices. Using electrophysiological evidence and anterograde and retrograde tract tracers, Romanski et al. (1999) find distinct connections between temporal and prefrontal cortices, as well as evidence of pathways supporting both feedforward and feedback information flow. This work suggests an anatomical framework that could underlie ventral and dorsal streams for audition.

In the visual system, the fundamental perceptual unit is a "visual object", which can be distinguished as physically distinct from other objects and the background. Identification of such an object is performed by computations along the ventral visual stream, which terminates in ventral regions of prefrontal cortex. Similarly, in hearing, sounds that we perceive as emanating from isolated physical sources are certainly distinct "auditory objects" (Bizley and Cohen 2013) (although it is likely that in human speech perception our fundamental units are much smaller, such as syllables (Binder et al. 2004)). In macaque, within the lateral belt surrounding primary auditory cor- 
tex, anterolateral regions, which differentiate among auditory objects, have anatomical connections that target ventral prefrontal areas (Romanski et al. 1999). Additionally, selective lesions of ventral prefrontal regions are found to impair performance on auditory discrimination tasks (i.e., identification of auditory objects) (Iversen and Mishkin 1970). Neurons in anterolateral regions also demonstrate the greatest specificity for various types of monkey calls (Rauschecker and Tian 2000), which are engaged during recognition of these auditory objects. These data suggest a ventral auditory path crucially engaged in the identification of auditory objects, analogous to the "what pathway", or ventral visual stream.

The dorsal visual stream, conversely, terminates in dorsal prefrontal regions and carries information relevant to sensorimotor ability, such as executing the appropriate hand shape and trajectory necessary to grasp a visualized object. Caudolateral regions of the auditory belt, adjacent to the primary auditory cortex, share synapses with caudal dorsolateral prefrontal cortex (DLPFC) (Romanski et al. 1999). DLPFC plays an essential role in visuospatial working memory; these connections suggest that it may play a similar role in spatial awareness within the auditory system. This assumption is supported by findings of activation in neurons in DLPFC during the localization of acoustic sound sources (Azuma and Suzuki 1984), in addition to studies revealing greater spatial selectivity of caudolateral auditory neurons (Rauschecker and Tian 2000), analogous to the visual "where/how pathway". Given the demonstrated homology between visual systems in macaque and human (Orban et al., 2004), as well as the superimposition of language systems upon shared evolutionary structures (Arbib and Bota 2003; Rauschecker and Scott 2009), it is appropriate that our investigation into the neurobiology of language has used data gathered from macaque research as a starting point for human investigation.

\section{Human neuroscience and the neurobiology of language}

While macaque physiology has informed our neurobiology of language, this field has been most strongly advanced through the recent development of high resolution structural and functional brain imaging methods, including fMRI, resting state magnetic resonance imaging (rsMRI), DTI, high-field electroencephalography (EEG), magnetoencephalography (MEG), electrocorticography (ECoG), and positron emission technology (PET), as well as 
targeted brain stimulation with transcranial magnetic stimulation (TMS) and transcranial direct current stimulation (tDCS). The availability of high resolution brain imaging has had a profound impact on neuroscientific advances by permitting noninvasive in vivo investigation of human physiology. Access, for the first time in history, to a methodologically rich human neuroscience has enabled the emergence of a true neurobiology of language. Previously untestable hypotheses about the mechanisms of human brain organization, and reorganization in the case of injury, can now be examined directly. This knowledge, and the ability to track these changes, will dramatically change the nature of the clinical neurosciences, including aphasia therapy. Thus far, human investigation supports and extends findings of dual streams originating in auditory cortices, as demonstrated in our macaque cousins and discussed in the following sections.

\section{Dual auditory streams}

Human research supports the dual stream hypothesis for hearing suggested by the nonhuman primate literature. Auditory objects, such as intelligible speech and speech-like sounds, activate anterolateral portions of the superior temporal cortices (Rauschecker, 2007) in human subjects, similar to the specificity of neurons in the anterolateral belt regions for monkey calls in the macaque (Rauschecker and Tian 2000). These data support the existence of a "what pathway" with selectivity for identifying meaningful auditory objects. Additional studies using fMRI and PET indicate that auditory spatial tasks activate temporal and parietal regions posterior to auditory cortex (analogous to the "where/how pathway") (Rauschecker 2007). Comparisons between anatomical tracer studies in macaque and brain imaging in humans confirm that structural connections are preserved across species for ventrolateral frontal regions associated with speech (Kelly et al. 2010). Such observations have led to the development of a unified dual stream model for human language, consistent with evidence from primate audition (Bornkessel-Schlesewsky et al., 2015).

\subsection{Dual streams: Speech perception}


The role of the dorsal visual stream in spatial awareness is understood as a means through which to perform a sensorimotor transformation in order to engage with, or avoid, an object (Shipp et al. 1998). In speech perception, the sensorimotor transformation enabled by the dorsal auditory stream has been interpreted as one that permits the listener to derive a motor plan in order to be able to produce what is heard (Rauschecker and Scott 2009). The mirror properties Buccino et al. find in human motor cortices for oral movements, described above, indicate shared neural mechanisms underlying both visual observation and production of speech (Buccino et al. 2001). Functional neuroimaging in the human suggests mirror characteristics in regions homologous to those found in macaque, including the dorsal aspect of pars opercularis of the inferior frontal gyrus (IFGop), inferior parietal lobule, and posterior superior temporal regions. These areas, associated with the dorsal auditory stream, demonstrate increasing activation when auditory speech signals are supplemented with visual stimuli showing talkers' faces (Skipper et al. 2005; Skipper et al. 2006), suggesting sensitivity of regions in the dorsal auditory stream to both auditory and visual input. If a function of the dorsal auditory stream is to support the production of perceived sounds, it seems reasonable that these regions would be closely tied to visually responsive areas that could further inform motor plans.

The role of the ventral auditory stream, in contrast, is to identify meaningful auditory objects. Hasson et al. (2007) use the so-called McGurkMacDonald effect (McGurk and MacDonald 1976) to test for neuronal populations sensitive to perceived speech that is detached from its sensory properties. The McGurk-McDonald syllable uses an auditory /pa/ paired with a visual $/ \mathrm{ka} /$, which is commonly perceived as the intermediary syllable $/ \mathrm{ta} /$, despite sharing no visual or auditory features with /ta/. The Hasson et al. study uses audiovisual presentation of the syllables $/ \mathrm{ka} /, / \mathrm{ta} /$, and $/ \mathrm{pa} /$ along with the McGurk-McDonald syllable. Taking advantage of the well-known phenomenon of repetition suppression (i.e., a decrease in neural activity for repeated processing of the same stimulus), the authors find two regions that exhibit lower activation for the processing of the McGurk-McDonald syllable, perceived as /ta/, when it followed an actual/ta/ syllable. This repetition suppression suggests that two regions of the left hemisphere - planum polare (anterior to primary auditory cortex) and a portion of the ventrally-located IFGop in the frontal lobe - are sensitive to the percept of stimuli, rather than low level sensory features, indicating a meaningful discrimination consistent with ventral stream processing. As increasingly complex information is pro- 
cessed at more expansive distances from primary sensory areas, such as primary visual and auditory cortices, planum polare and IFGop are two regions for which the complexity of this processing outpaces the original input, and a novel auditory object is produced through the integration of audiovisual information in the service of finding meaning. This identification of meaningful objects is the most representative function of the ventral streams.

\subsection{Dual streams: Language comprehension}

Both dorsal and ventral auditory streams play a decisive role in the understanding of speech and language in context that partially, but essentially, defines our neurobiology of language. The differential functions of these streams should now be clear. Additional distinctions between these streams include rate of maturation during typical development, as demonstrated by differences between children and adults in brain activation and connectivity while processing language in context.

Dick et al. (2010) contrast the neural bases underlying language comprehension in children (8-11 years) with adults, with all participants listening to the same stories during fMRI scanning. For both audio and audiovisual conditions, children show less activation overall, particularly in frontal and parietal regions of the right hemisphere. This suggests an asymmetrical developmental trajectory in which the right dorsal streams supporting visual and auditory processing are later to mature than ventral streams, which do not demonstrate such differences.

Dick et al. (2010) also find that the regions most affected by the addition of visual to auditory information are parts of the dorsal stream, including left posterior superior temporal gyrus (STGp)/sulcus (STSp) and supramarginal gyrus (SMG). Both children and adults demonstrate similar overall brain activation while listening to audiovisual stories, which engage regions associated with both dorsal and ventral streams. However, children demonstrate two differences of interest. The first is that they show activation in parts of the default mode network typically deactivated during attention to task, despite the fact that they are being engaged in a perceptual task during the acquisition of images. Secondly, in a network analysis comparing models of effective connectivity, children demonstrate weaker functional connections from IFGop to SMG, regions associated with the dorsal auditory stream. It should be noted that neither default mode activation nor limited effective connectivi- 
ty in the dorsal stream are observed in adults for audiovisual stimuli or in children for the isolated auditory signal. These weaker functional connections in children during audiovisual tasks suggest an ontogenetically later engagement of the dorsal auditory stream in multimodal language comprehension, with this ability likely developing during adolescence.

As referenced earlier in the context of finding a viable animal model for language, our present communication abilities have been superimposed on sensorimotor and neural systems that have emerged in the service of other functions over the course of the many epochs since we split from our last common ancestor shared with monkeys. This evolution most likely began with simple iconic manual gestures that provided a means to communicate with our conspecifics about concrete objects and events. Today, human speech is commonly accompanied not only by facial expression, but also by gestures, which often carry meaning above and beyond the verbal signal.

STSp is widely recognized as being generally responsive to any form of biological motion. On the whole, it is equivalently responsive to gestures regardless of whether or not they convey information. However, for adults, the effective connectivity of STSp with regions of the auditory dorsal stream, including planum temporale and SMG, is increased with the introduction of meaningful compared to meaningless gestures (Dick et al., 2012). This suggests a mediating role for STSp in the integration of auditory information with meaningful hand motion. Interestingly, this change in effective connectivity is not evident in children, and therefore is believed to develop later. These weaker connections in the dorsal stream, which were also noted in story comprehension tasks described above (Dick et al., 2010), indicate comparatively late maturation of dorsal connections. That we do not find any such differences for regions associated with the ventral stream suggests separate courses of development for the two streams, in addition to their distinct differences in anatomical connectivity and functional information processing.

\section{Conclusion}

As the new neurobiology of language continues to develop, built on a history of primate research and recent advances through the development of innovative tools in neuroimaging and statistical analysis, we find that a defining 
principle is the organization of a number of language functions around dorsal and ventral pathways. Language representations, such as they may be, are not housed in circumspect borders named after nineteenth century neurologists, but are instead bilateral and widely distributed across cortical and subcortical regions. The localizationalist models may be safely left behind; to the extent that we find one region tied to a given function, we may be certain that (i) it is participating in other functions and (ii) it is not operating independently. The fundamental unit of the neurobiology of language is no longer the region of interest, but the circuit.

For linguists, this means as much or as little as one wants it to. If one wishes to study language in isolation or in social spheres, there is no need to consider the neurobiology of language. However, if one is interested in understanding these linguistic phenomena - phenomena that evoked a level of fascination adequate to establish a career dedicated to their study - in a broader biological context, it is crucial to look outside of traditional linguistics and to embrace the contributions of interdisciplinary study. The examination of language with consideration of the three scholarly domains (linguistics, psychology, neurobiology) that inform our levels of investigation (computation, representation, implementation) provides the opportunity to advance our understanding of the complex human ability that unites our fields.

\section{References}

Agatonovic-Kustrin, S. and R. Beresford. 2000. "Basic concepts of artificial neural network (ANN) modeling and its application in pharmaceutical research". $J$ Pharm Biomed Anal 22. 717-727.

Arbib, M. and M. Bota. 2003. "Language evolution: Neural homologies and neuroinformatics". Neural Netw 16. 1237-1260.

Azuma, M. and H. Suzuki. 1984. "Properties and distribution of auditory neurons in the dorsolateral prefrontal cortex of the alert monkey". Brain Res 298. 343-346.

Binder, J.R., E. Liebenthal, E.T. Possing, D.A. Medler and B.D. Ward. 2004. "Neural correlates of sensory and decision processes in auditory object identification". Nat Neurosci 7. 295-301.

Binder, J.R., S.M. Rao, T.A. Hammeke, F.Z. Yetkin, A. Jesmanowicz, P.A. Bandettini, E.C. Wong, L.D. Estkowski, M.D. Goldstein, V.M. Haughton et al. 1994. "Functional magnetic resonance imaging of human auditory cortex". Ann Neurol 35. 662-672.

Bizley, J.K. and Y.E. Cohen. 2013. "The what, where and how of auditory-object perception". Nat Rev Neurosci 14. 693-707. 
Bornkessel-Schlesewsky, I., M. Schlesewsky, S.L. Small and J.P. Rauschecker. 2015. "Neurobiological roots of language in primate audition: Common computational properties". Trends Cogn Sci 19. 142-150.

Broca, P.P. 1861. "Nouvelle observation d'aphémie produite par une lésion de la moitié postérieure des deuxième et troisième circonvolutions frontales". Bulletin de la Société Anatomique de Paris 36. 398-407.

Buccino, G., F. Binkofski, G.R. Fink, L. Fadiga, L. Fogassi, V. Gallese, R.J. Seitz, K. Zilles, G. Rizzolatti, and H.J. Freund. 2001. "Action observation activates premotor and parietal areas in a somatotopic manner: An fMRI study". Eur $J$ Neurosci 13. 400-404.

Buchweitz, A., R.A. Mason, L.M. Tomitch and M.A. Just. 2009. "Brain activation for reading and listening comprehension: An fMRI study of modality effects and individual differences in language comprehension". Psychol Neurosci 2. 111123.

Bullmore, E. and O. Sporns. 2009. "Complex brain networks: graph theoretical analysis of structural and functional systems". Nat Rev Neurosci 10. 186-198.

Copeland, J. 1993. Artificial intelligence: A philosophical introduction. Hoboken: Wiley-Blackwell.

di Pellegrino, G., L. Fadiga, L. Fogassi, V. Gallese and G. Rizzolatti. 1992. "Understanding motor events: A neurophysiological study". Exp Brain Res 91. 176180.

Dick, A.S., S. Goldin-Meadow, A. Solodkin and S.L. Small. 2012. "Gesture in the developing brain". Dev Sci 15. 165-180.

Dick, A.S., A. Solodkin and S.L. Small. 2010. "Neural development of networks for audiovisual speech comprehension". Brain Lang 114. 101-114.

Doupe, A.J. and P.K. Kuhl. 1999. "Birdsong and human speech: Common themes and mechanisms". Annu Rev Neurosci 22. 567-631.

Déjerine, J.J. and A. Déjerine-Klumpke. 1895. Anatomie des centres nerveux. Paris: Rueff.

Ferrari, P.F., V. Gallese, G. Rizzolatti and L. Fogassi. 2003. "Mirror neurons responding to the observation of ingestive and communicative mouth actions in the monkey ventral premotor cortex". Eur J Neurosci 17. 1703-1714.

Freud, S. 1891. Zur Auffassung der Aphasie. Eine kritische Studie. Leipzig/Vienna: Deuticke.

Gall, F.J. 1825. Sur les fonctions du cerveau et sur celles de chacune de ses parties (vol. 6). Paris: J.B. Bailliére.

Gallese, V., L. Fadiga, L. Fogassi and G. Rizzolatti. 1996. "Action recognition in the premotor cortex". Brain 119 (pt. 2). 593-609.

Geschwind, N. 1970. "The organization of language and the brain". Science 170. 940-944.

Goni, J., M.P. van den Heuvel, A. Avena-Koenigsberger, N. Velez de Mendizabal, R. F. Betzel, A. Griffa, P. Hagmann, B. Corominas-Murtra, J.P. Thiran and O. Sporns. 2014. "Resting-brain functional connectivity predicted by analytic measures of network communication". Proc Natl Acad Sci U S A 111. 833-838.

Grashey, H. 1885. "Über Aphasie and ihre Beziehungen zur Wahrnehmung". Archiv für Psychiatrie und Nervenkrankheiten 16. 654-688. 
Hasson, U., J.I. Skipper, H.C. Nusbaum and S.L. Small. 2007. "Abstract coding of audiovisual speech: Beyond sensory representation”. Neuron 56. 1116-1126.

Iversen, S.D. and M. Mishkin. 1970. "Perseverative interference in monkeys following selective lesions of the inferior prefrontal convexity". Exp Brain Res 11. 376-386.

Kelly, C., L.Q. Uddin, Z. Shehzad, D.S. Margulies, F.X. Castellanos, M.P. Milham and M. Petrides. 2010. "Broca's region: Linking human brain functional connectivity data and non-human primate tracing anatomy studies”. Eur J Neurosci 32. 383-398.

Kiparsky, P. 1993. "Paninian linguistics". In: Asher, R. and J. Simpson (eds.), The encyclopedia of language and linguistics. Oxford: Pergamon Press. 2918-2923.

Levelt, W.J. 2001. "Spoken word production: A theory of lexical access". Proc Natl Acad Sci U S A 98. 13464-13471.

Lichtheim, L. 1885. "On aphasia”. Brain 7. 433-484.

Marr, D. 1982. Vision: A computational investigation into the human representation and processing of visual information. San Francisco, CA: W.H. Freeman.

McGurk, H. and J. MacDonald. 1976. "Hearing lips and seeing voices". Nature 264. 746-748.

Orban, G.A., D. Van Essen and W. Vanduffel. 2004. "Comparative mapping of higher visual areas in monkeys and humans". Trends Cogn Sci 8. 315-324.

Panini and S.C. Vasu. 1891. The Ashtadhyayi of Panini. (Translated into English by Srisa Chandra Vasu.) Benares: Sindhu Charan Bose, at the Panini Office.

Price, C.J. 2012. A review and synthesis of the first 20 years of PET and fMRI studies of heard speech, spoken language and reading". Neuroimage 62. 816847.

Rauschecker, J.P. 2007. "Cortical processing of auditory space: Pathways and plasticity". In: Mast, F. and L. Jäncke (eds.), Spatial processing in navigation, imagery and perception. New York: Springer. 389-410.

Rauschecker, J. P. and S.K. Scott. 2009. "Maps and streams in the auditory cortex: Nonhuman primates illuminate human speech processing". Nat Neurosci 12. $718-724$.

Rauschecker, J.P. and B. Tian. 2000. "Mechanisms and streams for processing of 'what' and 'where' in auditory cortex". Proc Natl Acad Sci U S A 97. 1180011806.

Romanski, L.M., B. Tian, J. Fritz, M. Mishkin, P.S. Goldman-Rakic and J.P. Rauschecker. 1999. "Dual streams of auditory afferents target multiple domains in the primate prefrontal cortex". Nat Neurosci 2. 1131-1136.

Rozzi, S., P.F. Ferrari, L. Bonini, G. Rizzolatti and L. Fogassi. 2008. "Functional organization of inferior parietal lobule convexity in the macaque monkey: Electrophysiological characterization of motor, sensory and mirror responses and their correlation with cytoarchitectonic areas". Eur J Neurosci 28. 1569-1588.

Shipp, S., M. Blanton and S. Zeki. 1998. "A visuo-somatomotor pathway through superior parietal cortex in the macaque monkey: Cortical connections of areas V6 and V6A". Eur J Neurosci 10. 3171-3193.

Skipper, J.I., H.C. Nusbaum and S.L. Small. 2005. "Listening to talking faces: Motor cortical activation during speech perception". Neuroimage 25. 76-89. 
Skipper, J.I., H.C. Nusbaum and S.L. Small. 2006. "Lending a helping hand to hearing: Another motor theory of speech perception". In: Arbib, M.A. (ed.), Action to language via the mirror neuron system. Cambridge: Cambridge University Press. 250-285.

Small, S.L., D.C. Noll, C.A. Perfetti, P. Hlustik, R. Wellington and W. Schneider. 1996. "Localizing the lexicon for reading aloud:replication of a PET study using fMRI". Neuroreport 7. 961-965.

Sporns, O., G. Tononi and R. Kotter. 2005. "The human connectome: A structural description of the human brain". PLoS Comput Biol 1. e42.

Tchernichovski, O. and G. Marcus. 2014. "Vocal learning beyond imitation: Mechanisms of adaptive vocal development in songbirds and human infants". Curr Opin Neurobiol 28. 42-47.

Tooby, J. and L. Cosmides. 2005. Evolutionary psychology: Conceptual foundations. Evolutionary psychology handbook. New York: Wiley.

Ungerleider, L.G., and M. Mishkin. 1982. "Two cortical visual systems". In: Ingle, D.J., M.A. Goodale and R.J.W. Mansfield (eds.), Analysis of visual behavior. Cambridge, CA: The MIT Press. 549-586.

Wernicke, C. 1874. Der Aphasische Symptomenkomplex. Breslau: Cohn \& Weigert.

Address for correspondence:

E. Susan Duncan

Department of Communication Sciences \& Disorders

Louisiana State University

68 Hatcher Hall

Field House Drive

Baton Rouge, LA 70803

United States of America

duncan1@1su.edu. 